\section{Rapidly worsening bulbar symptoms in a patient with spinobulbar muscular atrophy}

\author{
Montserrat Diaz-Abad, 1 Neil C. Porter² \\ 1Department of Medicine, Division of \\ Pulmonary and Critical Care Medicine, \\ 2Department of Neurology, Division of \\ Neuromuscular Diseases, University of \\ Maryland School of Medicine, Baltimore, \\ MD, USA
}

\begin{abstract}
X-linked spinobulbar muscular atrophy (Kennedy's disease) affects muscles and motor neurons, manifesting as weakness and wasting of bulbar, facial, and proximal limb muscles due to loss of anterior horn cells in the brain and spinal cord. We present the case of a patient with X-linked spinobulbar muscular atrophy with rapidly worsening bulbar symptoms caused by laryngopharyngeal irritation associated with a viral upper respiratory tract infection, seasonal allergies and laryngopharyngeal reflux, who dramatically improved with multimodality therapy.
\end{abstract}

\section{Introduction}

X-linked spinobulbar muscular atrophy (Kennedy's disease) is a neurodegenerative disease caused by an expansion of a polymorphic CAG tandem-repeat in the androgen receptor gene. It manifests as weakness and wasting of bulbar, facial, and proximal limb muscles due to loss of anterior horn cells in the brain and spinal cord. Age of onset is between 20-40 years with a slowly progressive course with normal or minimally reduced life span. ${ }^{1}$ We present the case of a patient with X-linked spinobulbar muscular atrophy with worsening bulbar symptoms caused by laryngopharyngeal irritation associated with a viral upper respiratory tract infection (URTI), seasonal allergies and laryngopharyngeal reflux, who dramatically improved with multimodality therapy.

\section{Case Report}

A $45 \mathrm{y} / 0$ man with a history of asthma and migraines presented to the neurology clinic in 2000 complaining of a 10 -year history of progressive muscle cramps that initially affected his jaws and forearms but subsequently involved his neck and all of four limbs. He also reported a 10-year history of tremors in his upper extremities that also worsened in severity over time. Family history was positive for tremors in his maternal aunt and 2 of his 3 brothers, and cramps in his third brother. Examination at the time of presentation was remarkable for obvious gynecomastia, mild periorbital facial weakness, possible wasting of the sternocleidomastoid muscles, fasciculations in multiple muscles of the face and arms, and a $10 \mathrm{~Hz}$ postural tremor in the arms. Of note, mental status, reflexes, strength, sensation and gait were all normal. Curiously, electrodiagnostic testing showed evidence of a generalized sensory neuropathy with absent left sural, median, ulnar and dorsal ulnar cutaneous nerve sensory responses and a slightly reduced superficial radial nerve sensory amplitude and conduction velocity. Electromyography showed evidence of acute denervation in the left lateral gastrocnemius and extensor digitorum brevis and evidence of chronic denervation in multiple muscles of the left upper and lower extremities. DNA testing for Kennedy's disease confirmed the diagnosis revealing an expansion of $47 \mathrm{CAG}$ repeats (normal being <33). The patient declined treatment for his tremor.

The patient continued to be seen in clinic on an annual basis, with slowly progressive disease with increasing difficulty climbing stairs and squatting. He was still able to continue working full time, however. At age 54 the patient reported new hoarseness and mild dysphagia which slowly worsened over the next year. He denied any heartburn or regurgitation. Videofluoroscopic evaluation of his swallowing revealed mild oropharyngeal dysphagia as well as evidence of esophageal reflux, but no laryngeal penetration or frank tracheal aspiration. Follow up swallowing evaluations were recommended every 3 to 6 months. The patient was subsequently seen at age 55 for an annual follow up visit, reporting increased difficulty climbing ladders and stairs. He additionally reported hoarseness after speaking for 5-10 minutes, persistent dysphagia and diffuse cramps.

Past medical history included mild intermittent asthma; hay fever with rhinitis and conjunctivitis during the summer; tonsillectomy; and migraines. No history of smoking. Medications were sumatriptan and acetaminophen/hydrocodone as needed and fluticasone nasal spray as needed during allergy season.

On physical exam he had fasciculations in the hand and tongue and with exertion in the face and platysmus muscles. There was temporal wasting and moderate gynecomastia noted. Voice was coarse, slightly nasal and weak, with moderate bilateral facial weakness present and cranial nerves otherwise intact. Reflexes were absent in the biceps, rest were $2+$. Strength was $4 / 5$ in the tongue and hip flexors; $4 \pm / 5$ in the deltoids, biceps, triceps; rest 5/5, and gait was
Correspondence: Montserrat Diaz-Abad, Sleep Disorders Center, Division of Pulmonary and Critical Care Medicine, University of Maryland School of Medicine, 685 West Baltimore Street, MSTF 800, Baltimore, MD 21201, USA.

Tel. +1.410.706.4771 - Fax: +1.410 .706 .0345$

E-mail: mdiaz@kunhardt.net

Key words: X-linked spinobulbar muscular atrophy, laryngopharyngeal reflux, allergy, laryngospasm, globus.

Contributions: MDA was involved with clinical care, interpretation of data, drafting the manuscript, revising the manuscript critically; NCP was involved with clinical care, interpretation of data, revising the manuscript critically.

Conflict of interests: the authors declare no potential conflict of interests.

Received for publication: 18 June 2013. Revision received: 3 July 2013.

Accepted for publication: 10 July 2013.

This work is licensed under a Creative Commons Attribution NonCommercial 3.0 License (CC BYNC 3.0).

(C) Copyright M. Diaz-Abad and N.C. Porter, 2013

Licensee PAGEPress, Italy

Neurology International 2013; 5:e21

doi:10.4081/ni.2013.e21

relatively normal. Body mass index was 24.5 $\mathrm{kg} / \mathrm{m}^{2}$.

The patient returned for an urgent visit, 3 months later, during allergy season. He reported a recent URTI with development of nasal congestion, rhinorrhea, severe post nasal drip, frequent throat clearing, globus pharyngeus, cough, dyspnea and wheezing. He had also experienced several episodes of sudden severe difficulty breathing, with throat tightness and audible stridor, which were very frightening and prompted two visits to the emergency department, where he was sent home after some improvement with prednisone and bronchodilators.

The patient was seen 2 weeks later at pulmonary clinic. He was feeling better, dyspnea and wheezing had resolved, but still reported mild residual nasal congestion, globus and frequent throat clearing. He was given a prescription for daily nasal fluticasone spray and cetirizine, with albuterol inhaler as needed. Pulmonary function tests (PFT) were ordered.

PFT values were within normal limits: forced expiratory volume in 1 second $\left(\mathrm{FEV}_{1}\right) 3.66 \mathrm{~L}$ (81\%), forced vital capacity (FVC) $2.92 \mathrm{~L}$ (89\%), $\mathrm{FEV}_{1} / \mathrm{FVC}$ ratio 80. Flow volume loop was abnormal, consistent with fixed airway obstruction (Figure 1). The patient developed laryngospasm with loud stridor and dyspnea during maximal expiratory pressure maneuvers. He was 
referred to Ears, Nose and Throat Clinic for airway inspection. Flexible fiberoptic nasolaryngoscopy showed no airway lesions or masses. Moderate erythema throughout the glottis and supraglottic larynx was noted, along with diminished abduction of the vocal cords. He was suspected of having laryngopharyngeal reflux and treated with esomeprazole, $40 \mathrm{mg}$ daily, along with behavior modification. He was seen 10 weeks later. Globus pharyngeus and throat clearing were almost gone, and the prior baseline chronic dysphagia had resolved and hoarseness had improved. He was eating more with increased appetite and was very satisfied with the response to therapy.

\section{Discussion}

We present the case of a patient with $\mathrm{X}$ linked spinobulbar muscular atrophy with worsening bulbar symptoms caused by laryngopharyngeal irritation associated with a viral URTI, seasonal allergies and laryngopharyngeal reflux who improved dramatically with multimodality therapy. X-linked spinobulbar muscular atrophy (Kennedy's disease) affects muscles and motor neurons, manifesting as weakness and wasting of bulbar, facial, and proximal limb muscles due to loss of anterior horn cells in the brain and spinal cord. As in this case, patients generally present in middle age with muscle cramps, bulbar weakness or leg weakness. ${ }^{2}$ Muscle weakness, wasting, and fasciculations are generally slowly progressive, with some patients becoming wheelchair-dependent 20 -30 years into their

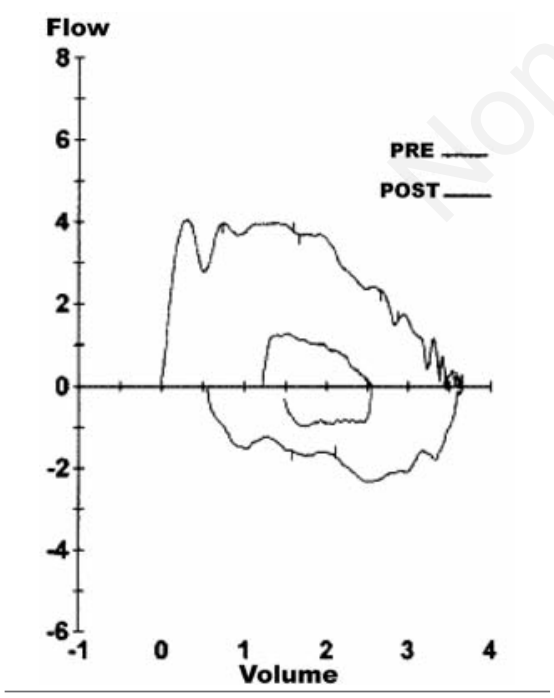

Figure 1. Flow volume loop on spirometry. There is flattening of the expiratory and inspiratory portions of the flow volume loop with decreased mid expiratory and mid inspiratory flows, consistent with fixed airway obstruction. course. ${ }^{3}$ Interestingly, in one large series of patients, nearly $100 \%$ of the patients showed abnormalities in their sensory responses on nerve conduction study. ${ }^{2}$ Dysphagia plays a prominent role in the morbidity represented from the disease. ${ }^{4}$ Unfortunately, there is no effective therapy for this condition; a recently concluded trial of dutasteride could not demonstrate a statistically significant effect on patient's muscle strength versus placebo. ${ }^{4}$ In the general population, patients who present with nonspecific pharyngeal and laryngeal symptoms are frequently considered to have laryngopharyngeal irritation related to extraesophageal reflux, also referred to as laryngopharyngeal reflux, which is caused by the retrograde movement of caustic gastric contents into the laryngopharynx causing mucosal inflammation. The most common presenting symptoms include hoarseness, dysphonia, globus, mild dysphagia, chronic cough, excessive throat clearing, dysphagia, postnasal drip and laryngospasm. Most patients do not have heartburn and regurgitation, the most common symptoms associated with gastroesophageal reflux disease (GERD). ${ }^{5}$

The diagnosis of laryngopharyngeal reflux is usually based on symptoms and findings on laryngoscopy, the most common being laryngeal erythema and edema, which are not specific. Because of this, the most accepted method to diagnose this condition in clinical practice is an empiric trial of a proton pump inhibitor (PPI) for 2-3 months. However, many patients with suspected laryngopharyngeal reflux do not respond to therapy and need to be evaluated for other potential causes of laryngopharyngeal irritation, including tobacco smoking, allergies, vocal cord overuse or abuse, infections, or postnasal discharge, and in many patients the etiology may be multifactorial. 5 Patients should be evaluated with all potential causes in mind to avoid unnecessary treatment and potential delay in diagnosis.

Morrison et al. ${ }^{6}$ described the irritable larynx syndrome, in which episodic laryngospasm and/or dysphonia, with/without globus or chronic cough occur. The authors hypothesize that these symptoms are triggered by a sensory stimulus when brainstem laryngeal neuronal networks are held in a hyperexcitable state and react inappropriately to sensory stimulation. Chronic reflux stimulation from GERD was felt to play a role in $>90 \%$ of patients and maximal acid suppression with PPI was considered necessary for reversal of the neural plastic changes to brainstem laryngeal control networks. In addition, of the 39 patients described, 17 associated onset of symptom to a viral illness, including 5 URTI and 3 flulike illnesses. ${ }^{6}$ Patients with spinobulbar muscular atrophy may be particularly susceptible to laryngospasm and other laryngeal symptoms caused by airway irritants leading to mucosal inflammation and damage to local sensory and motor neurons. Sperfeld et al. ${ }^{7}$ studied 49 patients with spinobulbar muscular atrophy and found recurrent laryngospasm in 23 (47\%) patients. In 10 of the 15 patients with laryngospasm who also had evidence of GERD, laryngospasm improved or resolved with antireflux therapy. All stages of the disease were represented, with a possible disease-specific vulnerability of a particular group of neurons involved in swallowing present in these patients. ${ }^{7}$ It is possible that some of the baseline bulbar symptoms such as dysphagia and hoarseness present in this patient were at least in part related to laryngopharyngeal reflux. The combined additional irritating effects of a URTI and seasonal allergies resulted in severe and distressing symptoms that prompted a more extensive evaluation and resulted in a very satisfactory response to specific therapy for these conditions.

\section{Conclusions}

While bulbar symptoms are common in patients with spinobulbar muscular atrophy, other disease processes that commonly produce bulbar symptoms in the general population may also be present in these patients, and a thorough evaluation for any potential treatable causes should be considered, in particular when symptoms are new or have rapidly worsened.

\section{References}

1. Finsterer J. Bulbar and spinal muscular atrophy (Kennedy's disease): a review. Eur J Neurol 2009;16:556-61.

2. Rhodes LE, Freeman BK, Auh S, et al. Clinical features of spinal and bulbar muscular Atrophy. Brain 2009;132:3242-51.

3. Kennedy WR, Alter M, Sung JH. Progressive proximal spinal and bulbar muscular atrophy of late onset. A sex-linked recessive trait. Neurology 1968;18: 671-80.

4. Fernández-Rhodes LE, Kokkinis AD, White $\mathrm{MJ}$, et al. A randomised, placebo-controlled trial of dutasteride in spinal and bulbar muscular atrophy. Lancet Neurol 2011;10: 140-7.

5. Vaezi MF. Laryngeal manifestations of gastroesophageal reflux disease. Curr Gastroenterol Rep 2008;10:271-7.

6. Morrison M, Rammage L, Emami AJ. The irritable larynx syndrome. J Voice 1999;13:447-55.

7. Sperfeld AD, Hanemann CO, Ludolph AC, Kassubek J. Laryngospasm: an underdiagnosed symptom of X-linked spinobulbar muscular atrophy. Neurology 2005;64:753-4. 